\title{
DESIGN, OPTIMIZATION AND IN VITRO CHARACTERIZATION OF SELF NANO EMULSIFYING DRUG DELIVERY SYSTEM OF OLMESARTAN MEDOXOMIL
}

\author{
SREENIVAS PATRO SISINTHY ${ }^{*}$, NALAMOLU KOTESWARA RAO² ${ }^{2}$ CHIN-YI LYNN SARAH ${ }^{1}$ \\ ${ }^{1,3}$ School of Pharmacy, Taylors University, Malaysia, ${ }^{2}$ School of Medicine, Taylors University, Malaysia \\ Email: sreenivaspatro.sisinthy@taylors.edu.my
}

Received: 05 Feb 2016 Revised and Accepted: 08 Nov 2016

\begin{abstract}
Objective: The objective of the present study was to design, optimise and characterise self nano emulsifying drug delivery systems (SNEDDS) for a poorly water soluble drug, olmesartan medoxomil (OLM) by Formulation by Design (FbD) approach with an aim to improve its solubility and dissolution.

Methods: The SNEDDS were systematically optimised using three factor Box-Behnken design. Concentration of formulation variables, namely, the oil phaseX1 (Capryol 90), the surfactant X2 (Cremophor EL), and the co-surfactant X3 (Transcutol P), was optimized for its impact on mean globule size (Y1), percentage drug release in $20 \mathrm{~min}$ (Y2) and turbidity (Y3) of the formulation. Ternary phase diagrams were constructed to select the areas of nanoemulsion and the amounts of oil, surfactant and cosurfactants as critical formulation variables. The prepared SNEDDS were characterised for globule size, dissolution studies, turbidity, and transmission electron microscopy (TEM).
\end{abstract}

Results: Following optimisation, the values of formulation variables were found to be $142.276 \mathrm{mg}$ (Capryol P), $399.999 \mathrm{mg}$ (Cremophor EL) and $598.871 \mathrm{mg}$ (Transcutol P) which produced a globule size of $12.64 \mathrm{~nm}$, percentage drug release of $93.34 \%$ and a turbidity of 0.02 FNU. TEM studies demonstrated spherical droplet morphology.

Conclusion: Thus, the present studies reveal that the SNEDDS is a promising drug delivery system approach for the enhancement of solubility and dissolution rate of OLM.

Keywords: Olmesartan, SNEDDS, Dissolution, Box-Behnken, Optimization

(c) 2017 The Authors. Published by Innovare Academic Sciences Pvt Ltd. This is an open access article under the CC BY license (http://creativecommons.org/licenses/by/4. 0/J DOI: http://dx.doi.org/10.22159/ijpps.2017v9i1.15166

\section{INTRODUCTION}

Olmesartan medoxomil (OLM), approved for use in the treatment of hypertension is a selective and competitive angiotensin-II receptor blocker [1]. Chemically OLM is (5-methyl-2-oxo-1,3-dioxol-4yl)methyl 5-(2-hydroxypropan-2-yl)-2-propyl-3-[[4-[2-(2H-tetrazol5 -yl)phenyl]phenyl]methyl]imidazole-4-carboxylate. It is introduced to the body as a prodrug and is hydrolyzed rapidly during gastrointestinal absorption by esterases found abundantly in the gastrointestinal tract, plasma and liver. OLM is de-esterified to form olmesartan, the active metabolite. The blood pressure lowering effect of OLM is dose dependent, causing vasodilation and retention of sodium. Results from a clinical trial conducted in hypertensive patients showed that OLM had exceptional pharmacological action with a good tolerance and no serious adverse effects. OLM also exhibited positive effects on liver disorders, atherosclerosis, and diabetic nephropathy. However, the oral bioavailability of OLM in healthy humans is only $26 \%$ due to its poor water solubility. OLM is highly lipophilic with a LogP of 5.55. It's mediocre bioavailability can also be contributed to the unfavourable breakage of the ester drug in GI fluids to olmesartan. Olmesartan, the parent molecule, has poor permeability with a $\log \mathrm{P}$ value of 1.2 at $\mathrm{pH}$ 7. Efflux pumps (P-glycoprotein) present in the GI tract also hamper the absorption of OLM [2, 3].

Self-emulsifying drug delivery system (SEDDS) is among the most promising methods to improve the solubility and oral bioavailability of hydrophobic drugs. It is an isotropic mixture that consists of oils, surfactants and co-surfactants that when used together in optimum concentrations, promote self-emulsification of the drug. When such formulations are diluted with the aqueous phase such as GI fluid, a fine, translucent to transparent oil-in-water $(\mathrm{o} / \mathrm{w})$ micro-or nanoemulsions formed upon mild agitation that is provided by the motility of the GI tract [4-6].

The micro-or nanoemulsions provide large interfacial surface areas that offer a considerable improvement in the rate and extent of oral absorption. Drug absorption is also increased by fluidising the intestinal membranes and hence, facilitating transcellular absorption. Furthermore, the opening of tight junctions facilitates paracellular transport. Drug absorption is also improved by the inhibition of efflux pumps such as P-gp [7, 8].

SMEDDS/SNEDDS, unlike regular emulsions, are stable preparations that have an increased interfacial surface area, inverse to its globule diameter. SMEDDS have a droplet size range of 100-250 nm and form optically clear to translucent dispersions. SNEDDS on the other hand, have a droplet size of less than $100 \mathrm{~nm}$. The appearance of SNEDDS dispersion is optically clear. A drug that has been formulated as an SMEDDS/ SNEDDS is dispersed as fine droplets in the gastrointestinal (GI) tract and this characteristic helps in improving the drug's dissolution profile and consequently, the drug's absorption and bioavailability. Drugs such as Cyclosporine A, Ritonavir, Saquinavir and a few others have been made commercially available as self-emulsifying systems. They are marketed as Neoral $₫$, Norvir $₫$ and Fortovase $₫$ respectively $[9,10]$.

These micro/nanoemulsions offer a considerable improvement in the rate and extent of oral absorption. The crucial step in the formulation of such system is in determining the appropriate oilsurfactant combination and individualised optimal ratio that can thoroughly dissolve the drug at its therapeutic concentration range.

The literature lacks any data about the optimisation of SNEDD for the improvement in OLM solubility and dissolution. Thus, the aim of this study was to design and optimisation of OLM-loaded SNEDDS containing surfactants reported to be bioenhancers. The box-behnken design was applied, and desirability function was used to optimize the concentration of oil, surfactant, and cosurfactant. As part of the optimisation process, the main effect, interaction effect and quadratic effects of amounts of oil, surfactant and co-surfactant on globule size, percentage drug release in $20 \mathrm{~min}$, and turbidity were investigated. The optimised formulation exhibiting promising in vitro drug dissolution is anticipated to improve oral absorption of the drug [11]. 


\section{MATERIALS AND METHODS}

\section{Materials}

Olmesartan medoxomil was purchased from Nivon Specialties (Mumbai,

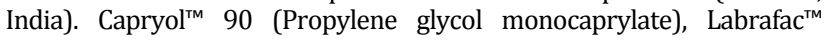
Lipophile WL 1349 (Caprylic/Capric triglyceride), Peceol ${ }^{\mathrm{TM}}$ (Glyceryl monooleate), Labrafil ${ }^{\circledR}$ M1944CS (Oleoyl macrogol-6 glycerides), Labrafac $^{\mathrm{TM}}$ PG (Propylene glycol dicaprylocaprate), Transcutol P and Maisine $^{\mathrm{TM}}$ 35-1 (Glycerol monolinoleate) were kindly supplied by Gattefosse SAS, Saint Priest, France as gift samples. Cremophor®EL (Macrogolglycerolricinoleate) used were from Sigma-Aldrich, BASF, Germany. PEG 400 (Poly (ethylene glycol)) and isopropyl myristate used were from Sigma-Aldrich, Missouri, USA. Tween $₫ 80$ (polysorbate 80) was obtained from RandM Chemicals (Essex, UK). Palm, sesame, sunflower, olive, castor and corn oil were obtained from ChemSoln (Selangor, Malaysia). Acetonitrile and potassium dihydrogen phosphate was purchased from Merck, Darmstadt, Germany. All other chemicals were of analytical grade and were used as received.

\section{Solubility studies}

Solubility studies were conducted by adding $50 \mathrm{mg}$ (excess) of OLM in $1 \mathrm{ml}$ of the vehicle to determine the solubility of OLM in various oils, surfactants and co-surfactants. The mixtures were vortexed using a vortex mixer (LMS, Mixer Uzusio, VTX-3000L) and kept in a water bath shaker (Julabo, TW20) at $50{ }^{\circ} \mathrm{C}$ for $48 \mathrm{~h}$ to allow the mixtures to equilibrate. After $48 \mathrm{~h}$, the supernatant was removed using a pipette and centrifuged (Hettich, Mikro $22 \mathrm{R}$ ) for $10 \mathrm{~min}$ at $4000 \mathrm{rpm}$ to sediment all the excess insoluble OLM.0.1 ml of the centrifuged supernatant was drawn up using a micropipette and was made up to $10 \mathrm{ml}$ with methanol. $1 \mathrm{ml}$ of the diluted sample was subsequently made up to $10 \mathrm{ml}$ with methanol for a total dilution factor of 1000 . The samples were then quantified using the HPLC method detailed below.

\section{HPLC method}

The quantitative estimation of OLM in the SNEDDS formulations and dissolution fluids was performed by HPLC. The HPLC system (Perkin Elmer, Flexar LC System) employed was equipped with a pump (Flexar FX-10), a diode array detector (Flexar PDA Plus), an autosampler (FX UHPLC Autosampler) and a data system (Chromera Chromatography Data System). Samples were separated by using a Brownlee Analytical Perkin ElmerC18 column. A modified HPLC method reported by Kumanan et al. is used in this study [12]. The mobile phase used was a mixture of Acetonitrile-0.05M Potassium dihydrogen phosphate adjusted to $\mathrm{pH} 3.0$ with orthophosphoric acid at a ratio of 50:50, v/v. The filtered (filtered through $0.45 \mu \mathrm{m}$ membrane filter) mobile phase components were pumped at a flow rate of $1.0 \mathrm{ml} / \mathrm{min}$. The column temperature of the system was maintained at $30^{\circ} \mathrm{C}$. The eluents were monitored at $256 \mathrm{~nm}$.

\section{Construction of ternary phase diagram}

Ternary phase diagrams are necessary to define the number and different types of phases formed. As the addition of OLM might interfere to a certain extent with the self-emulsification process, an alteration in the optimal oil-surfactant ratio might occur [13]. Hence, ternary phase diagrams were constructed using mixtures of the oil, surfactants and co-surfactant in different ratios to determine the optimal concentration of excipients required [14].

Oils studied were Capryol 90 and Maisine-35 and the surfactants employed were Tween 80 and Cremophor EL. Transcutol P was used as a co-surfactant. For all mixtures, the total of oil, surfactant, and cosurfactant amounts were always added to $100 \%$. The components in the mixtures were thoroughly mixed using a vortex mixer. The nanoemulsion formation efficiency of each formulation was assessed by adding $0.1 \mathrm{ml}$ of each mixture to $20 \mathrm{ml}$ of double distilled water in a conical flask.

The turbidimetric assessment was performed (Martini instruments, MI 415) and ternary plot diagrams were constructed using Pro Sim Ternary Diagram 1.0 free software to determine the region of self-emulsification. Only clear or slightly bluish tinged emulsions with droplet sizes lower than $200 \mathrm{~nm}$ were accepted as SMEDDS/SNEDDS [15].

\section{Preparation of OLM-SNEDDS formulations}

Based on the ternary phase diagrams, the oil, surfactant, and cosurfactant, chosen were Capryol 90, Cremophor EL, and Transcutol P respectively. OLM loaded SNEDDS formulations were prepared by adding OLM $(20 \mathrm{mg})$ to the blank formulations prepared with the various proportions of oil, surfactant and cosurfactant. OLM was dissolved by constant stirring and kept at $50{ }^{\circ} \mathrm{C}$ until reaching a transparent mixture. The preparation was stored at room temperature until further use for various in vitro characterizations.

\section{Experimental design}

A response surface methodology based on a three factor Box-Behnken design was used to develop and optimize the OLM formulations using Design Expert 9.0.6 software. The concentration of Capryol 90 (Factor X1), Cremophor EL (Factor X2), and Transcutol P (Factor X3) were varied from 100 to $300 \mathrm{mg}, 200$ to $400 \mathrm{mg}$, and 300 to $600 \mathrm{mg}$ respectively. The independent factors and dependent variables employed in this design are shown in table 1 . The effects of the independent factors on the dependable variables (Y1: droplet size of diluted SMEDDS, Y2: percentage drug release in $20 \mathrm{~min}$ and Y3: turbidity) were studied. A total of 17 experiments were designed by the employed software with 5 centre points. Experiments were run in random order to increase the predictability of the model. Optimisation was performed using a desirability function to obtain the levels of X1, X2 and X3, which minimised (Y1) and (Y3) and maximised (Y2) [16-21]. The quadratic model generated by design is as follows:

$$
\begin{gathered}
Y_{1}=b_{0}+b_{1} X 1+b_{2} X 2+b_{3} X 3+b_{12} X 1 X 2+b_{13} X 1 X 3+b_{23} X 2 X 3+ \\
b_{11} X 1^{2}+b_{22} X 2^{2}+b_{33} X 3^{2}----(1)
\end{gathered}
$$

The above equation comprises the coefficient of the intercept, first order main effect (X1, X2, X3), interaction terms (X1X2, X1,X3, $\mathrm{X} 2, \mathrm{X} 3)$, and higher order effect $\left(\mathrm{X}^{2}{ }^{2}, \mathrm{X}^{2}, \mathrm{X}^{2}\right)$, where $\mathrm{Y}$ is the measured response; response variables selected for the optimization purpose were globule size, $\%$ drug release in $20 \mathrm{~min}$ and turbidity.

Table 1: Variables used in the Box-behnken design

\begin{tabular}{lll}
\hline & Levels & Medium (0) \\
\cline { 2 - 3 } Independent variables & Low (-1) & 200 \\
X1: amount of oil (mg) & 100 & 300 \\
X2: amount of surfactant (mg) & 200 & 450 \\
X3: amount of co-surfactant (mg) & 300 & 400 \\
Dependent variables & Constraints & 600 \\
Y1: droplet size (nm) & Minimize & \\
Y2: \% OLM released in 20 min & Maximize & \\
Y3: turbidity (FNU) & Minimize & \\
\hline
\end{tabular}

\section{Characterization of OLM-loaded snedds}

\section{Droplet size analysis}

Droplet size measurement is an important property in assessing the self-emulsification performance. The droplet size affects the rate and extent of drug release and the stability of the emulsion.
Several techniques are commonly used to determine the droplet size distributions of the emulsion. These techniques include Photon Correlation Spectroscopy (PCS), Laser Diffraction and Coulter Counter. The droplet size of the emulsion is thought to be a crucial element in the self-emulsification performance as it could influence the rate and extent of drug release as well as its oral absorption. It is assumed that 
the droplet size should be as fine as possible. The reduction of droplet size to values below $100 \mathrm{~nm}$ has led to the formation of SNEDDS, which are stable, isotropic and clear $\mathrm{o} / \mathrm{w}$ dispersions.

The droplet size of the 17 Box-Behnken formulations was analysed using a Malvern Zetasizer, (Nano ZS). A $0.1 \mathrm{ml}$ from each formulation was diluted to $20 \mathrm{ml}$ with purified water at $25{ }^{\circ} \mathrm{C}$ and the contents were gently stirred using a magnetic stirrer. The droplet size of the resultant emulsions was determined by photon correlation spectroscopy using a Zetasizer Nano ZS (Malvern Instruments, UK). A laser beam at $632 \mathrm{~nm}$ wavelength was used, and light scattering was monitored at $25^{\circ} \mathrm{C}$ at a $173^{\circ}$ angle [22].

\section{Visual observations and turbidity}

The self-emulsifying property of the mixtures was assessed by their visual appearance. The emulsion should either be visually clear to lightly turbid if SMEDDS are formed or clear to slightly bluish if SNEDDS are formed. Emulsions that are dull and greyish white were not accepted and the presence of large oil droplets indicates poor emulsification. From each formulation, $0.1 \mathrm{ml}$ was introduced into $20 \mathrm{ml}$ of double distilled water at room temperature and the contents were gently stirred manually. The turbidity of the resultant emulsions was recorded in Formazin Nephelometric Unit (FNU) using Martini instruments, MI 415 [23]. The final appearance of each emulsion produced was also observed and noted.

\section{Dissolution studies}

17 Box-Behnken design formulations containing $20 \mathrm{mg}$ of OLM were prepared prior to dissolution. The prepared formulations were filled into size 00 hard gelatine capsules and held to the bottom of the vessel using stainless steel sinkers [24]. The in vitro dissolution behaviours of OLM tablet $(20 \mathrm{mg})$ and the 17 BoxBehnken SMEDDS formulations were assessed using the USP rotating paddle Electrolab Dissolution Tester (TDT-08L). $900 \mathrm{ml}$ of $0.1 \mathrm{~N} \mathrm{HCl}$ was first prepared by diluting concentrated $\mathrm{HCl}$ in a volumetric flask. The dissolution media was heated at $37 \pm 0.5^{\circ} \mathrm{C}$ and the rotating speed was maintained at $50 \mathrm{rpm}$. OLM-loaded SMEDDS were placed into the media. At predetermined time intervals of 5, 10, $15,20,30,45$, and $60 \mathrm{~min}, 5 \mathrm{ml}$ aliquots were collected and replaced with an equal amount of fresh dissolution media to maintain sink conditions. The samples collected were filtered using a $0.45 \mathrm{~mm}$ Millipore nylon filter and were analysed using HPLC at $\lambda=256 \mathrm{~nm}$. The release profiles from OLM-loaded SNEDDS were compared to the release profile of OLM marketed tablets.

\section{Transmission electron microscopy}

OLM-loaded SNEDDS were evaluated using transmission electron microscopy to examine their morphology and structure. A Zeiss 902 CEM microscope (Zeiss, Barcelona, Spain) was used for measurement. The sample was diluted with distilled water $(1: 200)$ and thoroughly mixed by gentle shaking. One sample droplet was deposited on a copper grid and the excess was absorbed using a filter paper. Subsequently, the grid was inverted and stained with one drop of $1 \%$ phosphor-tungstic acid (PTA) for 10s. Excess PTA was removed, and examination of the grid was done at $60-80 \mathrm{kV}$ [25].

\section{RESULTS AND DISCUSSION}

\section{Solubility studies}

SNEDDS consists of a mixture of oil, surfactants, co-surfactants, and drug. When introduced to an aqueous phase, the mixture should form a clear, monophasic liquid at room temperature and should have good solvent properties that allow the drug to be present in solubilized form. The solubility of OLM in various vehicles is shown in fig. 1. Amongst the various oily phases that are screened, Capryol ${ }^{\mathrm{TM}} 90$ and Maisine ${ }^{\mathrm{TM}}$ 351 demonstrated the highest solubility with OLM and were chosen for further investigations. Two surfactants, namely Cremophor ${ }^{\circledR}$ EL and Tween ${ }^{\circledR} 80$ has shown the excellent solubilizing ability for OLM. Transcutol P, an absorption enhancer and solubilizer, was found to be very efficient in solubilizing OLM. Therefore, it was chosen as a cosurfactant in the development of OLM-SNEDD formulations that aimed to improve drug loading capabilities.

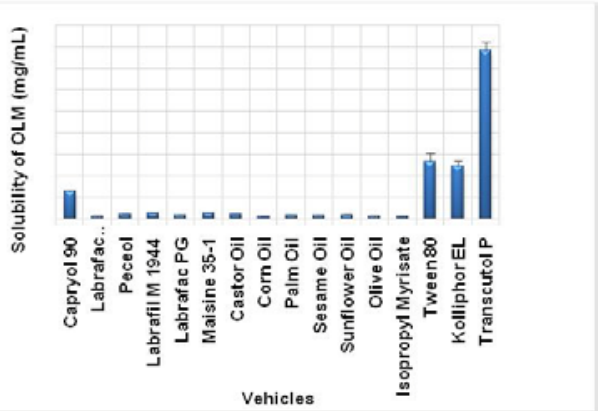

Fig. 1: Solubility studies of OLM in different vehicles. Each value represents mean $\pm \operatorname{SD}(n=3)$
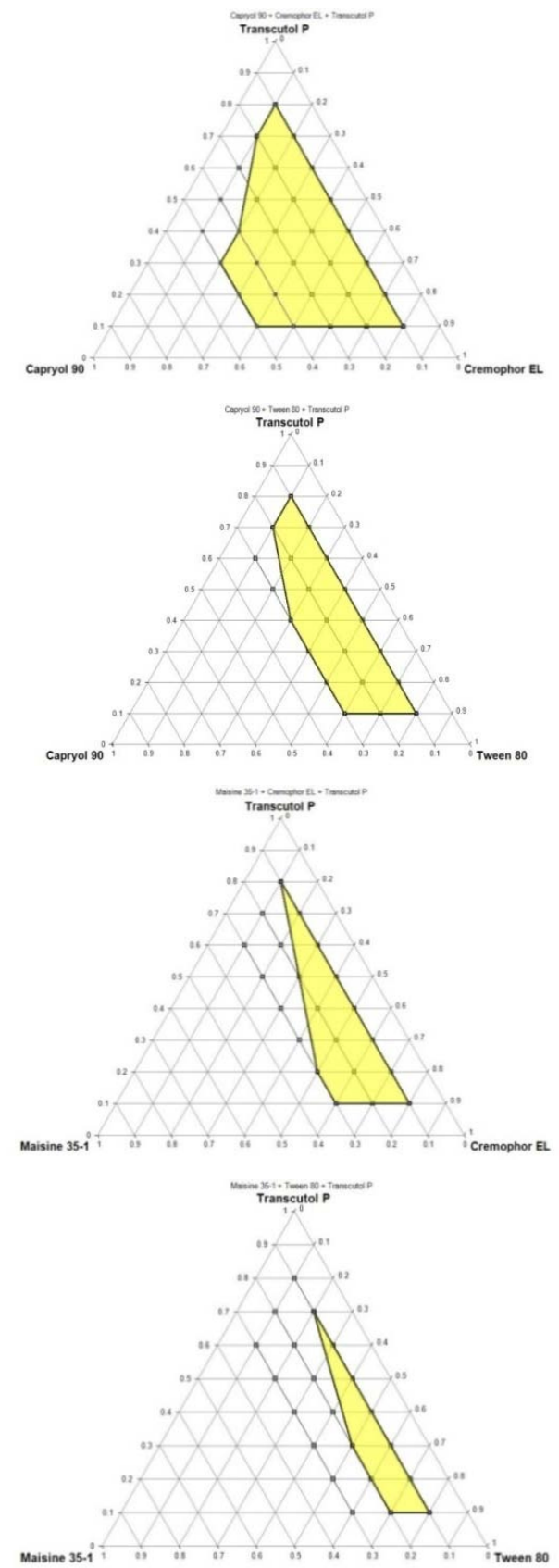

Fig. 2: Ternary phase diagram for combination of (a) Capryol 90 Cremophor EL, and Transcutol P (b)Capryol 90, Tween 80, and Transcutol P(c) Maisine 35-1, Cremophor EL, and Transcutol P

(d) Maisine 35-1, Tween 80, and Transcutol $P$ 


\section{Construction of ternary phase diagram}

Based on results obtained through preliminary screenings, four ternary phase diagram formulations were prepared. The system I: Capryol $^{\mathrm{TM}}$ 90/Cremophor EL/Transcutol P; system II: Capryol ${ }^{\mathrm{TM}}$ 90/Tween 80/Transcutol P; system III: Maisine ${ }^{\mathrm{TM}}$ 35-1/ Cremophor ${ }^{\circledR}$ EL/Transcutol P; system IV: Maisine ${ }^{\mathrm{TM}} 35-1 /$ Tween ${ }^{\circledR}$ 80/Transcutol P. The phase diagrams were depicted in fig. 2 (a-d). The shaded regions indicate nanoemulsion region. A wider region of nanoemulsion indicates better self nano emulsifying ability.

The ternary phase diagrams obtained showed that systems I (fig. 2(a)) and II (fig. 2(b)) exhibited wider nano emulsification regions as compared to systems III (fig. 2(c)) and IV (fig. 2(d)). This indicates that system I and II had better self-nano emulsification properties than that of the systems III and IV. Systems I and II contained Capryol ${ }^{\mathrm{TM}}$ 90and yielded nanoemulsions containing as high as $30-50 \%$ oily phase composition. On the other hand systems III and IV, containing Maisine ${ }^{T M}$ 35-1, produced nanoemulsions till a maximum oil concentration of $20-30 \%$ only. Thus, Capryol ${ }^{\mathrm{TM}} 90$ was selected for the formulation of OLM-loaded SNEDDS using Cremophor EL.

\section{Optimisation and evaluation of olmesartan Snedds}

Seventeen formulations were prepared and analysed as per the BoxBehnken experimental design. The constraints used in this study were globule size, $\%$ drug released in $20 \mathrm{~min}$ and turbidity. The constraints applied were to minimise the globule size and turbidity and to maximise the \% drug release in $20 \mathrm{~min}$. The experimental runs and the observed responses were given in table 2 . The independent and response variables were related using the polynomial equation with statistical analysis through DesignExpert ${ }^{\circledR}$ software 9.0.6. The values of the coefficients X1, X2 and X3 are related to the effect of these variables on the response. A positive sign of coefficient indicates a synergistic effect while a negative term indicates an antagonistic effect upon the response [26, 27]. The larger coefficient means the independent variable has a more potent influence on the response.

The mathematical relationship in the form of factors' coefficients and its corresponding p-values for the measured responses are listed in table 3. Coefficients with a p-value less than 0.05 had a significant effect on the prediction efficiency of the model for the measured response.

\section{Mean globule size}

All the batches have shown a globule size for less than $100 \mathrm{~nm}$ ranging from $12.7 \mathrm{~nm}$ to $89.01 \mathrm{~nm}$. Regression analysis for response Y1 (mean globule size) suggested a quadratic model and the cubic model was aliased due to insufficient design points (table3). ANOVA data suggested the regression be significant $(\mathrm{p}<0.0001)$. The polynomial equation (2) for mean globule size proposed by the model is as follows:

$$
\begin{aligned}
\mathrm{Y} 1= & 27.13+28.39 \mathrm{X} 1-5.73 \mathrm{X} 2-2.55 \mathrm{X} 3-5.4 \mathrm{X} 1 \mathrm{X} 2-1.4 \mathrm{X} 1 \mathrm{X} 3- \\
& 0.66 \mathrm{X} 2 \mathrm{X} 3+14.94 \mathrm{X}^{2}+4.3 \mathrm{X}^{2}-2.4 \mathrm{X}^{2}-----(2)
\end{aligned}
$$

Synergistic effects of $\mathrm{X} 1, \mathrm{X} 1^{2}$ and $\mathrm{X} 2^{2}$ and antagonistic effects of $\mathrm{X} 2$, $\mathrm{X} 3, \mathrm{X} 1 \mathrm{X} 2, \mathrm{X} 1 \mathrm{X} 3, \mathrm{X} 2 \mathrm{X} 3$ and $\mathrm{X}^{3}$ on $\mathrm{Y} 1$ were observed. Mean globule size was lowest in Batch 8 at low levels of oil, mid-level of surfactant and high level of co-surfactant (table 2). Form table4, it can be seen that Y1 (droplet size) was significantly affected by the antagonistic effect of the amount of surfactant (X2) and the interaction effect $\mathrm{X} 1 \mathrm{X} 2$ (between the amount of oil and surfactant) with $p$-values of 0.0017 and 0.0133 respectively.

\begin{tabular}{|c|c|c|c|c|c|c|}
\hline \multirow[t]{2}{*}{ Batch } & \multicolumn{3}{|c|}{ Independent variables } & \multicolumn{3}{|c|}{ Dependent variables } \\
\hline & Capryol 90 (mg) & Cremophor EL (mg) & Transcutol P (mg) & Globule size (nm) & \% Drug Release in $20 \mathrm{~min}$ & Turbidity (FNU) \\
\hline 1 & 200 & 400 & 600 & 20.59 & 92.34 & 7.34 \\
\hline 2 & 200 & 200 & 300 & 36.12 & 88.45 & 17.22 \\
\hline 3 & 300 & 400 & 450 & 65.78 & 81.57 & 41.38 \\
\hline 4 & 200 & 200 & 600 & 32.43 & 91.12 & 14.39 \\
\hline 5 & 300 & 200 & 450 & 89.01 & 74.87 & 46.22 \\
\hline 6 & 200 & 300 & 450 & 27.66 & 88.35 & 16.48 \\
\hline 7 & 200 & 300 & 450 & 29.35 & 89.78 & 12.55 \\
\hline 8 & 100 & 300 & 600 & 12.7 & 96.55 & 0 \\
\hline 9 & 200 & 300 & 450 & 28.88 & 89.23 & 14.66 \\
\hline 10 & 300 & 300 & 600 & 61.43 & 79.48 & 41.44 \\
\hline 11 & 200 & 300 & 450 & 25.12 & 86.34 & 14.87 \\
\hline 12 & 300 & 300 & 300 & 69.42 & 76.97 & 44.85 \\
\hline 13 & 200 & 300 & 450 & 24.65 & 87.93 & 17.37 \\
\hline 14 & 100 & 300 & 300 & 15.08 & 97.76 & 0 \\
\hline 15 & 100 & 400 & 450 & 14.55 & 98.77 & 0 \\
\hline 16 & 100 & 200 & 450 & 16.16 & 95.67 & 0 \\
\hline 17 & 200 & 400 & 300 & 26.93 & 90.78 & 12.56 \\
\hline
\end{tabular}

Table 2: Experimental design and observed responses from Box-Behnken design

\begin{tabular}{|c|c|c|c|c|c|}
\hline Response & Model & Std. Dev. & Predicted $\mathrm{R}^{2}$ & Adjusted R ${ }^{2}$ & Remarks \\
\hline \multirow[t]{4}{*}{ Mean globule size } & Linear & 9.84 & 0.7115 & 0.8069 & \\
\hline & $2 \mathrm{Fl}$ & 10.64 & 0.4407 & 0.7742 & \\
\hline & Quadratic & 3.28 & 0.8827 & 0.9785 & Suggested \\
\hline & Cubic & 2.15 & & 0.9908 & Aliased \\
\hline \multirow[t]{4}{*}{$\%$ drug release in $20 \mathrm{~min}$} & Linear & 1.84 & 0.8924 & 0.9315 & Suggested \\
\hline & $2 \mathrm{Fl}$ & 1.92 & 0.7969 & 0.9251 & \\
\hline & Quadratic & 1.36 & 0.8653 & 0.9624 & Suggested \\
\hline & Cubic & 1.33 & & 0.9644 & Aliased \\
\hline \multirow[t]{4}{*}{ Turbidity } & Linear & 4.71 & 0.8644 & 0.9140 & \\
\hline & $2 \mathrm{Fl}$ & 5.27 & 0.6983 & 0.8921 & \\
\hline & Quadratic & 1.80 & 0.9606 & 0.9875 & Suggested \\
\hline & Cubic & 1.86 & & & \\
\hline
\end{tabular}

Table 3: Regression analysis of mean globule size, \% drug released in $20 \mathrm{~min}$ and turbidity 


\section{Percent drug release in $\mathbf{2 0}$ min}

Regression analysis for response Y2 (\% drug release in $20 \mathrm{~min}$ ) suggested a linear and quadratic model and the cubic model was aliased due to insufficient design points (table 3). ANOVA data suggested the regression be significant $(\mathrm{p}<0.0001)$. The polynomial equation proposed by the model for \% drug release in $20 \mathrm{~min}$ (Y2) is:

$$
\begin{aligned}
\mathrm{Y} 2= & 90.47-5.37 \mathrm{X} 1+1.32 \mathrm{X} 2+1.51 \mathrm{X} 3-0.15 \mathrm{X} 1 \mathrm{X} 2+1.05 \mathrm{X} 1 \mathrm{X} 3- \\
& 0.027 \mathrm{X} 2 \mathrm{X} 3-8.851^{2}+0.16 \mathrm{X}^{2}+0.3 \mathrm{X}^{2}----(3)
\end{aligned}
$$

Synergistic effects of X2, X3, X1 X3, X22 and X32 and antagonistic effects of X1, X1X2, X2X3 and X12 on Y2 were observed. Percent drug release was highest in Batch 15 at low levels of oil, high level of surfactant and mid-level of co-surfactant and lowest in Batch 5 at high levels of oil, low level of surfactant and mid-level of co-surfactant.
From the table 4, it can be seen that Y2 (\% drug released in $20 \mathrm{~min}$ ) was significantly affected by the antagonistic effect of the amount of surfactant (X2) with a $p$-value of 0.01 . The possible explanation for this is that the amount of surfactant was mainly responsible for the increase in the cumulative percentage of drug release from the formulations.

The increase in cumulative drug release was mainly attributed to rapid self-emulsification of the formulations due to instantaneous dispersion in the medium after the dissolution of the capsule shell [28]. The presence of surfactant-assisted the formation of $0 / \mathrm{W}$ droplets and rapid spreading of the formulation in the aqueous media. This increases the water penetration of oil droplets, resulting in disruption of the interface and thereby decreasing the droplet size and eventually increasing the release rate [29].

\begin{tabular}{|c|c|c|c|c|c|c|c|c|c|c|}
\hline & Intercept & $\mathbf{X 1}$ & $\mathbf{X} 2$ & X3 & $\mathbf{X} 1 X 2$ & X1X3 & $\mathrm{X} 2 \mathrm{X} 3$ & $\mathbf{X} 1^{2}$ & $X 2^{2}$ & $\mathbf{X} 3^{2}$ \\
\hline Globule size & 27.132 & 28.3938 & -5.73375 & -2.55 & -5.405 & -1.4025 & -0.6625 & 14.941 & 4.3015 & -2.416 \\
\hline $\mathrm{P}$ & & $<0.0001$ & 0.0017 & 0.0641 & 0.0133 & 0.4212 & 0.6986 & $<0.0001$ & 0.0312 & 0.1748 \\
\hline \% Drug Release in $20 \mathrm{~min}$ & 90.466 & -5.37 & 1.31875 & 1.50625 & -0.15 & 1.05 & -0.0275 & -8.85175 & 0.15575 & 0.30075 \\
\hline $\mathrm{P}$ & & $<0.0001$ & 0.0952 & 0.0635 & 0.8811 & 0.3136 & 0.9781 & $<0.0001$ & 0.8734 & 0.759 \\
\hline Turbidity & 15.186 & 21.7363 & -2.06875 & -1.4325 & -1.21 & -0.8525 & -0.5975 & 7.7045 & -0.9905 & -1.318 \\
\hline $\mathrm{p}$ & & $<0.0001$ & 0.0139 & 0.0587 & 0.2198 & 0.3740 & 0.5271 & $<0.0001$ & 0.2950 & 0.1758 \\
\hline
\end{tabular}

Table 4: Regression coefficients for the responses

\section{Turbidity}

Regression analysis for response Y3 (turbidity) suggested a quadratic model and the cubic model was aliased due to insufficient design points (table 3). ANOVA data suggested the regression to be significant $(\mathrm{p}<0.0001)$. The polynomial equation proposed by the model for turbidity (Y3) is:

$$
\begin{gathered}
\mathrm{Y} 3= \\
15.19+21.74 \mathrm{X} 1-2.07 \mathrm{X} 2-1.43 \mathrm{X} 3-1.21 \mathrm{X} 1 \mathrm{X} 2-0.85 \mathrm{X} 1 \mathrm{X} 3- \\
0.59 \mathrm{X} 2 \mathrm{X} 3+7.7 \mathrm{X}^{2}-0.99 \mathrm{X}^{2}-1.32 \mathrm{X}^{2}----(4)
\end{gathered}
$$

Synergistic effects of $\mathrm{X} 1$ and $\mathrm{X} 1^{2}$ and antagonistic effects of $\mathrm{X} 2, \mathrm{X} 3$, $\mathrm{X} 1 \mathrm{X} 2, \mathrm{X} 1 \mathrm{X} 3, \mathrm{X} 2 \mathrm{X} 3, \mathrm{X}^{2}$ and $\mathrm{X} 3^{3}$ on $\mathrm{Y} 3$ were observed. Turbidity was lowest in all the formulations with low oil content whereas it was found highest in Batch 5 at high levels of oil, low level of surfactant and mid-level of co-surfactant. Turbidity value depends significantly on the globule size and thus can be used indirectly to reflect globule size. By analysing these coefficients in the regression equation, it can be said that turbidity is distinctly influenced by oil and the surfactant concentration as seen in table 4. As fig. 4(c) shows, the increase in surfactant concentration reduces the turbidity indicating reduced globule size. This might be due to the surfactant-induced reduction in surface tension between the aqueous phase and organic phase. In addition, surfactant helps to stabilize the newly generated surfaces and prevents particle aggregation. The increase in oil concentration increases the turbidity indicating the increase in globule size. The fact that the size of oil droplets is highly dependent on its concentration can be explained in terms of the tendency of the oil droplets to coalesce at high oil concentration

\section{Response surface and contour plot analysis}

The relationship between the dependent and independent variables was further elucidated using contour and response surface plots. These types of plots are very useful for studying the interaction effects between the two factors for understanding how the effect of one factor will be influenced by the change in the level of another factor as shown in fig. 3 (a-c). As these types of plots can only express two independent variables at a time against the response, one independent variable must always be fixed [30].

\section{Identification and evaluation of optimum formulation using the desirability function}

For the analysis of experiments with multiple responses, desirability function technique is used where several responses have to be optimised simultaneously.

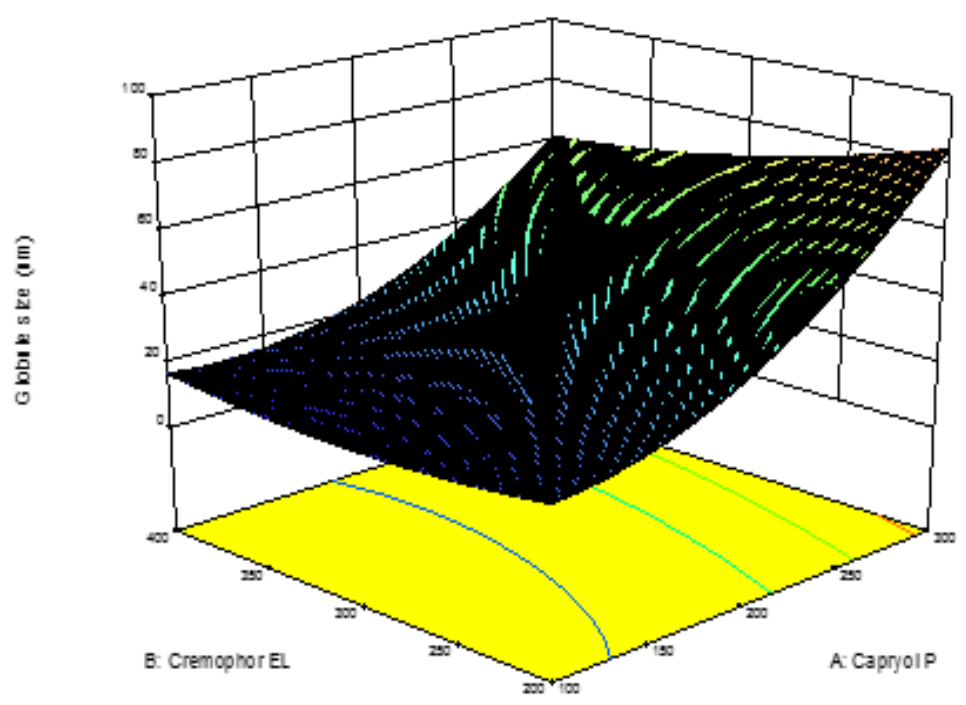

(a) 


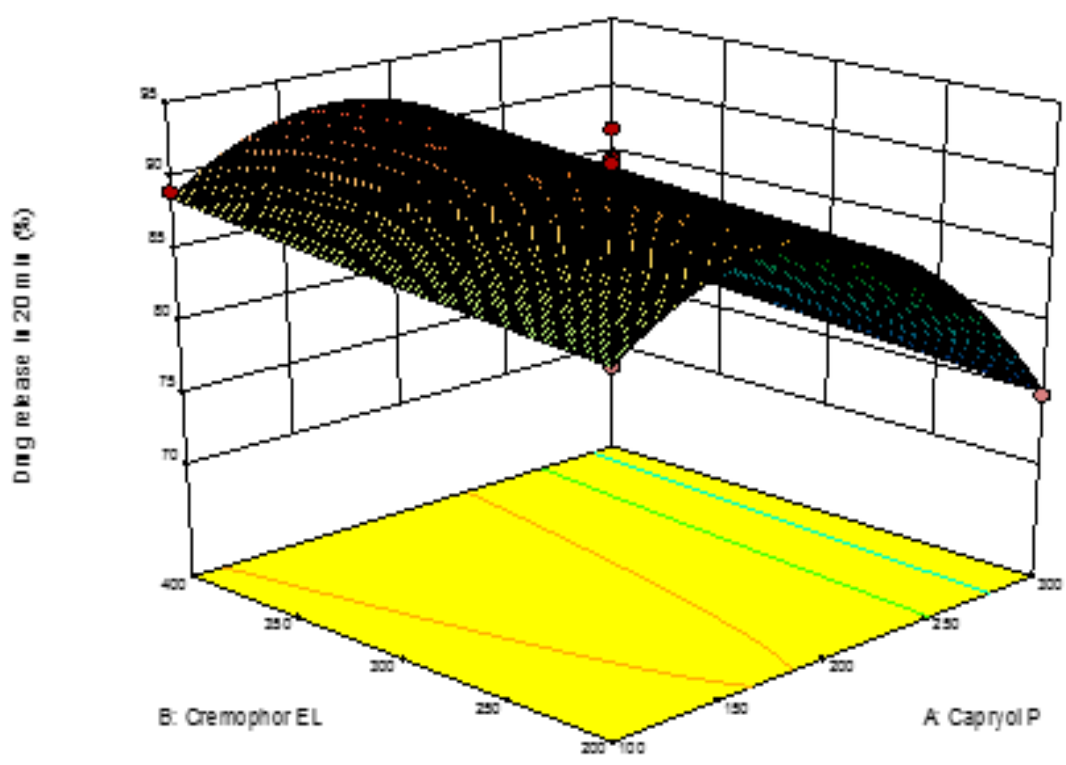

(b)

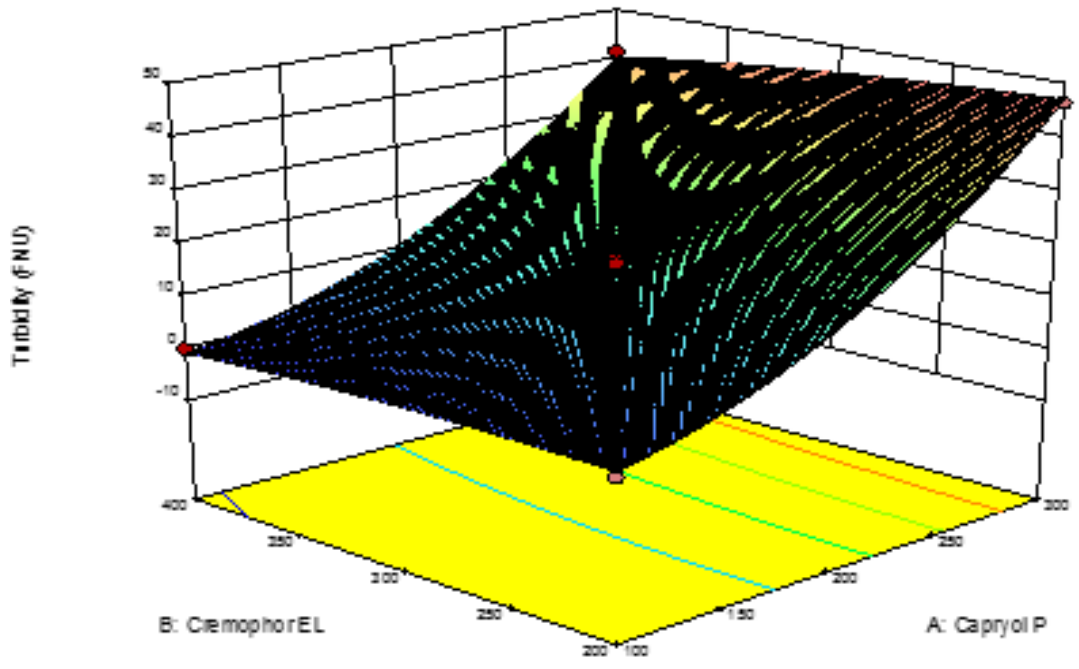

(c)

Fig. 3: Response surface plots of interaction of Capryol $P$ and Cremophor EL on (a) globule size; (b) \% drug release in 20 min; (c) turbidity

OLM formulation with a composition of $142.276 \mathrm{mg}$ of Capryol 90 (oil), $399.996 \mathrm{mg}$ of Cremophor EL (surfactant) and $598.913 \mathrm{mg}$ of Transcutol P (co-surfactant) was observed to be optimal, in terms of desired globule size (minimum), percent drug release in 20 min (maximum) and turbidity (minimum). Fig. 4(a) shows the highest desirability (0.978) and fig. 4(b) shows the overlay plots with optimum globule size (12.64 nm), percent drug release in $20 \mathrm{~min}(93.4 \%)$ and turbidity $(0.02 \mathrm{FNU})$.

In this case, $\mathrm{Y} 1$ and $\mathrm{Y} 3$ were set to be minimised whereas $\mathrm{Y} 2$ was set to be maximised. The desirability function $\mathrm{D}$, over the experimental domain, was calculated by Design Expert (9.0.6) software. The scale of desirability function ranges between $\mathrm{D}=0$, for a completely undesirable response and $D=1$, if the response is at the most desirable value.

\section{In vitro dissolution studies}

In vitro dissolution studies were carried out in $0.1 \mathrm{~N} \mathrm{HCl}$. The dissolution performance of the optimised SNEDDS was compared with that of the marketed product. The release profiles are presented in fig. 5. The percentage drug release for the optimised OLM SNEDDS was found to be $98.4 \%$ in 20 min whereas it was only $28.3 \%$ for the marketed formulation in $20 \mathrm{~min}$.
The faster dissolution from the SNEDDS formulation can be attributed to the fact that, the drug is insolubilized form in the formulation and upon exposure to the dissolution medium it results in the formation of smaller droplets that can dissolve rapidly in the dissolution medium.

\section{Transmission electron microscopy}

Morphological and structural examination of the optimised OLMloaded SNEDDS formulation was carried out using transmission electron microscopy. TEM images post-dilution showed that spherical micelles were formed (fig. 6).

These results were according to DLS results with no signs of coalescence confirming the efficiency of the nanoemulsion preparation method used. 


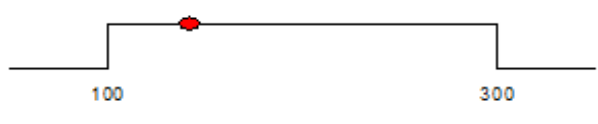

A: Capryol P $=142.276$

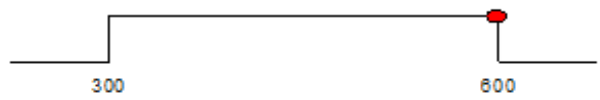

C: Transcutol $P=598.87$

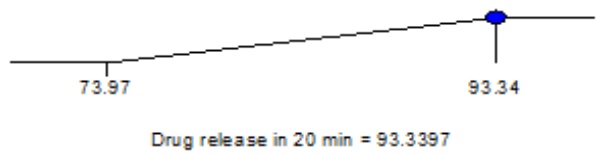

Desirability $=0.978$

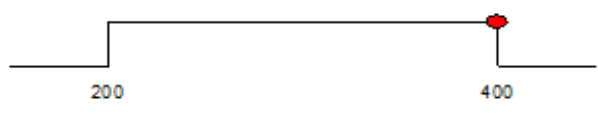

B:Cremophor EL $=399.999$

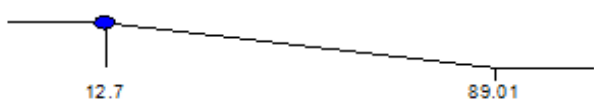

Globule size $=12.6437$

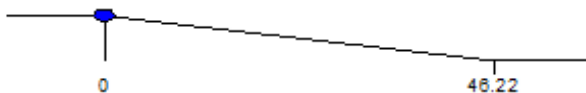

T urbidity $=0.0208436$

(a)

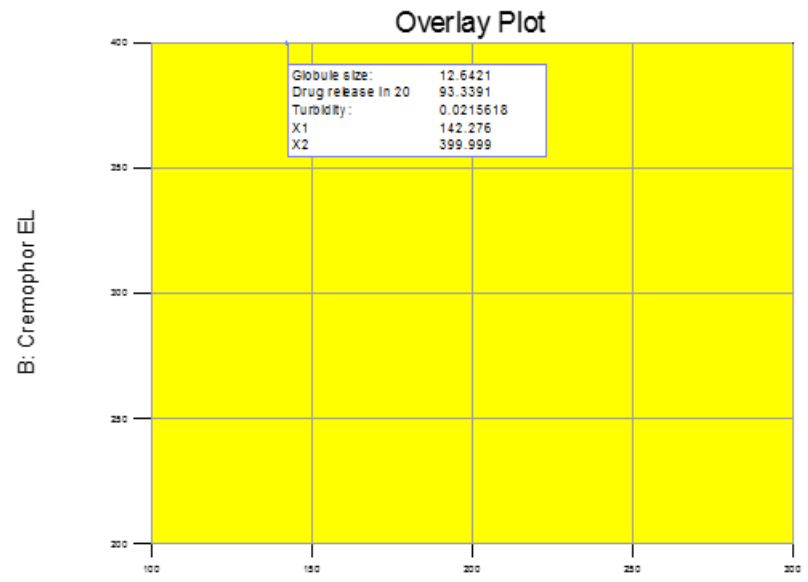

A. Capryol P

(b)

Fig. 4: (a) Optimized results using desirability data; (b) Overlay plot for the optimization of Capryol 90, Cremophor RH 40 and Transcutol P.

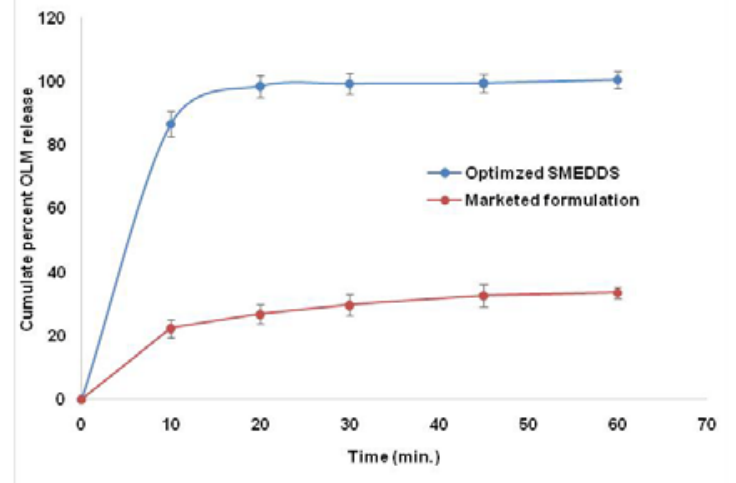

Fig. 5: In vitro dissolution profiles of optimized OLM SNEDDS and the marketed formulation $(n=3)$

The nanoemulsion droplets emerged as dark and the surroundings were found to be bright. No signs of drug precipitation were observed inferring the stability of the formed nanoemulsion. Closer analysis of
TEM images reveals that each globule is surrounded by a thick layer indicating the formation of monolayer around the emulsion droplets, reducing the interfacial energy, and forming a barrier to coalescence.

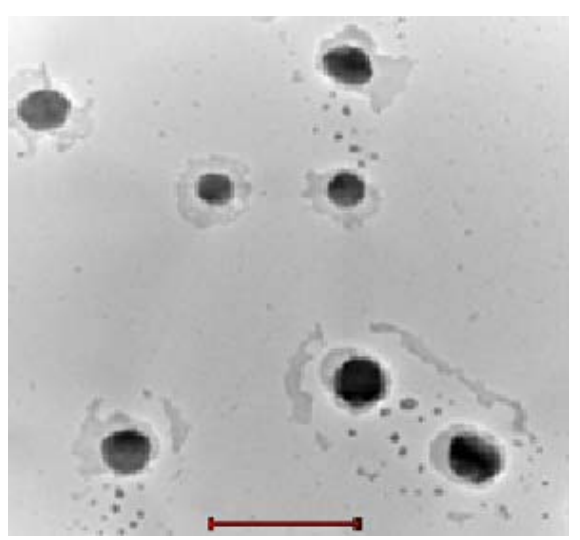

Fig. 6: TEM of optimized olmesartan SNEDDS formulation (Bar length 50 nm) 
Transmission electron microscopy of F3 revealed dark and spherical spots against a light background and the droplet size revealed by TEM was in conformity with the zeta sizing results. Although NE is one of the finest modes of delivery for hydrophobic therapeutic agent OLM, but due to liquid nature of the dosage form, it is normally associated with transportation issues.

\section{CONCLUSION}

The design and optimisation of OLM SNEDDS formulation were carried out by Box-Behnken design-response surface methodology combined with desirability function. The effect of the amount of oil, surfactant and co-surfactant were investigated for their influence on globule size, percentage drug release in $20 \mathrm{~min}$ and turbidity. The optimised formulation consisted of $142.276 \mathrm{mg}$ of Capryol 90, $399.999 \mathrm{mg}$ of Cremophor EL and $598.871 \mathrm{mg}$ of Transcutol $\mathrm{P}$ which could provide a globule size of $12.64 \mathrm{~nm}, 93.34 \%$ of drug release in $20 \mathrm{~min}$ and a turbidity of 0.02 FNU. The optimised SNEDDS formulation exhibited a 3fold enhancement in dissolution rate as evident from in vitro dissolution studies. Thus, the present study illustrates the potential use of SNEDDS formulation approach for the improvement of solubility and dissolution rate of the poorly soluble drug, Olmesartan Medoxomil.

\section{ACKNOWLEDGEMENT}

The work was supported and funded by Taylor's Research Grant Scheme (TRGS/ERFS/1/2013/SOP/003), Taylor's University, Malaysia. The authors wish to acknowledge Gattefosse, Saint-Priest, France for providing gift samples of different oils, surfactants and cosurfactants and also Stat-ease for providing us with Design Expert 9.0.6 Trail software.

\section{CONFLICT OF INTERESTS}

\section{Declared none}

\section{REFERENCES}

1. Chabukswar AR, Kuchekar BS, Jagdale SC, Mehetre DM, More AS, Lokhande PD. Development and validation of an RP-HPLC method for simultaneous estimation of olmesartan medoxomil and amlodipine besylate in tablet dosage form. Arch Appl Sci Res 2010;2:307-12.

2. Raval C, Joshi N, Patel J, Upadhyay UM. Enhanced oral bioavailability of olmesartan by using novel solid self-emulsifying drug delivery system. Int J Adv Pharm 2012;2:82-92.

3. Kang MJ, Kim HS, Jeon HS, Park JH, Lee BS, Ahn BK, et al. In situ intestinal permeability and in vivo absorption characteristics of olmesartan medoxomil in self-micro emulsifying drug delivery system. Drug Dev Ind Pharm 2012;38:587-96.

4. Khade S, Pore Y. Formulation and evaluation of neusilin US2 adsorbed amorphous solid self-micro emulsifying delivery system of atorvastatin calcium. Asian J Pharm Clin Res 2016;9:93-100.

5. Date AA, Desai N, Dixit R, Nagarsenker M. Self-nanoemulsifying drug delivery systems: formulation insights, applications and advances. Nanomed 2010;5:1595-616.

6. Kallakunta VR, Bandari S, Jukanti R, Veerareddy PR. Oral selfemulsifying powder of lercanidipine hydrochloride: formulation and evaluation. J Powder Technol 2012;221:375-82.

7. Narang AS, Delmarre D, Gao D. Stable drug encapsulation in micelles and microemulsions. Int J Pharm 2007;345:9-25.

8. Gursoy RN, Benita S. Self-emulsifying drug delivery systems (SEDDS) for improved oral delivery of lipophilic drugs. Biomed Pharmacother 2004;58:173-82.

9. Anand S, Gupta R, Prajapati SK. Self-micro emulsifying drug delivery system. Asian J Pharm Clin Res 2016;9:1-6.

10. Nasr A, Gardouh A, Ghoniam H, Abdelghany E, Ghorab M. Effect of oils, surfactants and cosurfactants on phase behavior and physicochemical properties of self-nanoemulsifying drug delivery system (SNEDDS) for irbesartan and olmesartan. Int J Appl Pharm 2016;8:13-24.

11. Basalious EB, Shawky N, Badr-Eldin SM. SNEDDS containing bioenhancers for improvement of dissolution and oral absorption of lacidipine: I. Development and optimization. Int J Pharm 2010;391:203-11.

12. Kumanan R, Jitendra MR, Manasa R. Stability indicating RPHPLC method development and validation of olmesartan medoxomil. Asian J Pharm Biol Res 2011;1:79-86.
13. Singh B, Khurana L, Bandyopadhay S, Kapil R, Katare 00. Development of optimized self-nanoemulsifying drug delivery systems (SNEDDS) of carvedilol with enhanced bioavailability potential. Drug Delivery 2011;18:599-612.

14. Date AA, Nagarsenker MS. Design and evaluation of selfnanoemulsifying drug delivery systems (SNEDDS) for cefpodoxime proxetil. Int J Pharm 2007;329:166-72.

15. Baek MK, Lee JH, Cho YO, Kim HH, Lee GW. Selfmicroemulsifying drug-delivery system for improved oral bioavailability of pranlukast hemihydrate: preparation and evaluation. Int J Nanomed 2013;8:167-76.

16. Palamakula A, Nutan MHT, Khan MA. Response surface methodology for optimization and characterization of Limonene-based coenzyme Q10 self nanoemulsified capsule dosage form. AAPS PharmSciTech 2004;5:114-21.

17. Bahloul B, Lassoued MA, Souad S. A novel approach for the development and optimization of self emulsifying drug delivery system using HLB and response surface methodology: application to fenofibrate encapsulation. Int J Pharm 2014; 466:341-8.

18. Pawar YB, Purohit H, Valicherla GR, Munjal B, Lale SV, Patel SB, et al. Novel lipid based oral formulation of curcumin: development and optimization by design of experiments approach. Int J Pharm 2012;436:617-23.

19. Jain AK, Thanki K, Jain S. Solidified self-nanoemulsifying formulation for oral delivery of combinatorial therapeutic regimen: part I. Formulation development, statistical optimization, and in vitro characterization. Pharm Res 2014;31:923-45.

20. Beg S, Swain S, Singh HP, Patra ChN, Rao ME. Development, optimization, and characterization of solid selfnanoemulsifying drug delivery systems of valsartan using porous carriers. AAPS PharmSciTech 2012;13:1416-27.

21. Singh S, Pathak K, Bali V. Product development studies on surface-adsorbed nanoemulsion of olmesartan medoxomil as a capsular dosage form. AAPS PharmSciTech 2012;13:1212-21.

22. Luykx DM, Peters RJ, van Ruth SM, Bouwmeester H. A review of analytical methods for the identification and characterization of nano delivery systems in food. J Agric Food Chem 2008;56:8231-47.

23. Taha EI. Development and characterization of new indomethacin self-nanoemulsifying formulations. Sci Pharm 2009;77:443-51.

24. Richard AS, James MH, Tod FJ, Myles S. Effects of sinker shapes on dissolution profiles. J Pharm Sci 2006;78:35-9.

25. Villar AMS, Naveros BC, Campmany ACC, Trenchs MA, Rocabert CB, Bellowa LH. Design and optimization of selfnanoemulsifying drug delivery systems (SNEDDS) for enhanced dissolution of gemfibrozil. Int J Pharm 2012. 431:161-75.

26. Patel J, Patel A, Raval M, Sheth N. Formulation and development of a self-nanoemulsifying drug delivery system of irbesartan. J Adv Pharm Technol Res 2011;2:9-16.

27. Huang YB, Tsai YH, Lee SH, Chang JS, Wu PC. Optimization of $\mathrm{pH}$-dependent release of nicardipine hydrochloride extendedrelease matrix tablets using response surface methodology. Int J Pharm 2005;289:87-95.

28. Balakrishnan P, Lee BJ, Oh DH, Kim JO, Lee YI, Kim DD, et al. Enhanced oral bioavailability of coenzyme Q10 by selfemulsifying drug delivery systems. Int J Pharm 2009; 374:66-72.

29. Marasini N, Yan YD, Poudel BK, Choi HG, Yong CS, Kim JO. Development and optimization of self-nanoemulsifying drug delivery system with enhanced bioavailability by boxbehnken design and desirability function. J Pharm Sci 2012;101:4584-96.

30. Wu XG, Li G, Gao YL. Optimization of the preparation of nalmefene-loaded sustained-release microspheres using central composite design. Chem Pharm Bull 2006;54:977-81.

\section{How to cite this article}

- Sreenivas Patro Sisinthy, Nalamolu Koteswara Rao, Chin Yi Lynn Sarah. Design, optimization and in vitro characterization of self nano emulsifying drug delivery system of olmesartan medoxomil. Int J Pharm Pharm Sci 2017;9(1):94-101. 